Phys. Rev. B 81, 115416 (2010); Phys. Rev. B 101, 049909(E) (2020)

\title{
Magnetism of Finite Graphene Samples: Mean-Field Theory compared with Exact Diagonalization and Quantum Monte Carlo Simulation
}

\author{
Hélène Feldner, ${ }^{1}$ Zi Yang Meng, ${ }^{2}$ Andreas Honecker, ${ }^{3}$ Daniel Cabra, ${ }^{1}$ Stefan Wessel, ${ }^{2}$ and Fakher F. Assaad ${ }^{4}$ \\ ${ }^{1}$ Institut de Physique et Chimie des Matériaux de Strasbourg, UMR7504, \\ CNRS-UdS, 23 rue du Loess, BP43, 67034 Strasbourg Cedex 2, France \\ ${ }^{2}$ Institut für Theoretische Physik, Universität Stuttgart, 70550 Stuttgart, Germany \\ ${ }^{3}$ Institut für Theoretische Physik, Georg-August-Universität Göttingen, \\ Friedrich-Hund-Platz 1, 37077 Göttingen, Germany \\ ${ }^{4}$ Institut für Theoretische Physik und Astrophysik, \\ Universität Würzburg, Am Hubland, 97074 Würzburg, Germany \\ (Dated: October 28, 2009; revised January 15, 2010; corrected January 5, 2020)
}

\begin{abstract}
The magnetic properties of graphene on finite geometries are studied using a self-consistent meanfield theory of the Hubbard model. This approach is known to predict ferromagnetic edge states close to the zig-zag edges in single-layer graphene quantum dots and nanoribbons. In order to assess the accuracy of this method, we perform complementary exact diagonalization and quantum Monte Carlo simulations. We observe good quantitative agreement for all quantities investigated provided that the Coulomb interaction is not too strong.

PACS numbers: $71.10 . \mathrm{Fd} ; 73.22 . \mathrm{Pr} ; 75.40 . \mathrm{Mg}$
\end{abstract}

\section{INTRODUCTION}

Graphene consists of a single layer of carbon atoms arranged in a honeycomb crystal lattice ${ }^{1}$ and is a promising material with unique electronic properties. Among the most important characteristics, one should mention the presence of massless carriers, weak spin-orbit coupling, insensitivity to an external electrostatic potential (Klein paradox), fractional quantum Hall effect, etc. (for a review of the main features of graphene see Ref. 2). The electronic properties of graphene nanostructures such as nanoribbons or quantum dots are expected to be very different from bulk graphene. In fact, the Coulomb interaction is considerably enhanced in smaller geometries such as quantum dots, leading for example to unusual blockade effects. ${ }^{3-5}$ On the other hand, the edge effect, which depends strongly on the geometry of the sample boundary, modifies the electronic structure of graphene. ${ }^{6-8}$ In particular, it has been predicted that finite graphene samples can exhibit magnetic edge states (see, e.g., Refs. 9-19) suggesting potential spintronics applications of graphene nanodevices. ${ }^{20}$

It is common practice to use a mean-field theory (MFT) of the Hubbard model to investigate the magnetic properties of graphene in finite geometries (see, e.g., Refs. 9,10,13-19). Such a MFT is applicable to any interaction and any geometry in a quite economic way: within the self-consistent mean-field (MF) approximation the main numerical effort is to solve the single-electron problem on a finite lattice. However, as far as we are aware, very little is known about the accuracy of this approximation.

The main purpose of the present paper is to address this issue and check the accuracy of the MFT. We start by recalling a real-space formulation of the MFT in Sec. II. In Sec. III we briefly look at periodic boundary conditions ${ }^{21}$ and show that we can reproduce edge- ferromagnetism for a dot with zig-zag edges. ${ }^{14,15,19}$ The accuracy of the MFT is carefully examined in Sec. IV where we present a comparison with exact diagonalization for a small "dot" and quantum Monte Carlo (QMC) simulations on a larger system with periodic boundary conditions. We conclude with a summary and perspectives in Sec. V.

\section{MODEL AND COMPUTATION}

Since we are interested in the magnetic properties of graphene, interactions should be taken into account. To this end, we study the Hubbard model whose Hamiltonian reads

$$
H=-t \sum_{\langle i, j\rangle, \sigma} c_{i, \sigma}^{\dagger} c_{j, \sigma}+U \sum_{i} n_{i, \uparrow} n_{i, \downarrow}
$$

with $n_{i, \sigma}=c_{i, \sigma}^{\dagger} c_{i, \sigma} .\langle i, j\rangle$ are nearest neighbors on a honeycomb lattice. We denote the total number of sites by $N$ and the number of electrons with a spin projection $\sigma=\uparrow, \downarrow$ by $N_{\sigma}$.

Due to the exponential growth of the Hilbert space dimension with $N$, a direct exact diagonalization of the Hubbard model (2.1) at half filling is only possible for system sizes until about 20 sites. In order to deal with larger system sizes we use a MF approximation:

$$
\begin{aligned}
H^{M F}= & -t \sum_{\langle i, j\rangle, \sigma} c_{i, \sigma}^{\dagger} c_{j, \sigma} \\
& +U \sum_{i}\left(\left\langle n_{i, \uparrow}\right\rangle n_{i, \downarrow}+n_{i, \uparrow}\left\langle n_{i, \downarrow}\right\rangle-\left\langle n_{i, \uparrow}\right\rangle\left\langle n_{i, \downarrow}\right\rangle\right) .
\end{aligned}
$$

It should be noted that the MF approximation breaks the $\mathrm{SU}(2)$-symmetry of the original Hubbard model (2.1). 
We compute the ground state

$$
|G S\rangle=\prod_{\alpha \leq N_{\uparrow}} d_{\uparrow \alpha}^{\dagger} \prod_{\beta \leq N_{\downarrow}} d_{\downarrow \beta}^{\dagger}|0\rangle, \quad d_{\sigma, \alpha}=\sum_{i} Q_{\sigma, \alpha i}^{*} c_{\sigma, i}
$$

and the one-electron spectrum $\epsilon_{\sigma, \alpha}$ of the MF Hamiltonian (2.2) using the LAPACK library.

This yields the ground-state energy, the local density

$$
\left\langle n_{\sigma, i}\right\rangle=\sum_{\alpha \leq N_{\sigma}} Q_{\sigma, \alpha i}^{*} Q_{\sigma, i \alpha}
$$

the local magnetization $\left\langle S_{i}^{z}\right\rangle=\frac{1}{2}\left\langle n_{i, \uparrow}-n_{i, \downarrow}\right\rangle$, as well as the spin correlation functions

$$
\begin{aligned}
&\left\langle S_{i}^{z} S_{j}^{z}\right\rangle=\frac{1}{4}\left(\sum_{\sigma=\uparrow, \downarrow} \sum_{\alpha=1}^{N_{\sigma}} \sum_{\beta \neq \alpha}^{N_{\sigma}} Q_{\sigma, j \alpha} Q_{\sigma, i \beta}\right. \\
& \quad \times\left\{Q_{\sigma, \alpha j}^{*} Q_{\sigma, \beta i}^{*}-Q_{\sigma, \beta j}^{*} Q_{\sigma, \alpha i}^{*}\right\} \\
&\left.\quad-\left\langle n_{j \uparrow}\right\rangle\left\langle n_{i \downarrow}\right\rangle-\left\langle n_{j \downarrow}\right\rangle\left\langle n_{i \uparrow}\right\rangle\right) \\
&\left\langle S_{i}^{x} S_{j}^{x}\right\rangle=\left\langle S_{i}^{y} S_{j}^{y}\right\rangle=-\frac{1}{4}\left(\sum_{\alpha=1}^{N_{\uparrow}} \sum_{\beta=1}^{N_{\downarrow}} Q_{\uparrow, j \alpha} Q_{\downarrow, i \beta} Q_{\downarrow, \beta j}^{*} Q_{\uparrow, \alpha i}^{*}\right. \\
&\left.\quad+Q_{\uparrow, i \alpha} Q_{\downarrow, j \beta} Q_{\downarrow, \beta i}^{*} Q_{\uparrow, \alpha j}^{*}\right)
\end{aligned}
$$

for $i \neq j$ and

$$
\begin{aligned}
\left\langle S_{i}^{z} S_{j}^{z}\right\rangle & =\left\langle S_{i}^{x} S_{j}^{x}\right\rangle=\left\langle S_{i}^{y} S_{j}^{y}\right\rangle \\
& =\frac{1}{4}\left(\left\langle n_{i \uparrow}\right\rangle+\left\langle n_{i \downarrow}\right\rangle-2\left\langle n_{i \uparrow}\right\rangle\left\langle n_{i \downarrow}\right\rangle\right)
\end{aligned}
$$

for $i=j$.

Self-consistency requires that the expectation values $\left\langle n_{\sigma, i}\right\rangle$ entering (2.2) are equal to the expression (2.4) derived from this Hamiltonian. We solve this condition iteratively using suitable initial conditions with given numbers of electrons $N_{\sigma}$. To overcome convergence problems, we use a thermal state compatible with the Fermi-Dirac distribution at a given temperature instead of the ground state for the first iterations. ${ }^{22}$ In this case the average density is computed as

$$
\left\langle n_{\sigma, i}\right\rangle=\sum_{\alpha \in \Omega_{\sigma}} Q_{\sigma, \alpha i}^{*} Q_{\sigma, i \alpha},
$$

where $\Omega_{\sigma}$ is a set of $N_{\sigma}$ single-particle states chosen randomly with probability $n\left(\epsilon_{\sigma, \alpha}\right)=1 /\left(1+e^{\left[\beta\left(\epsilon_{\sigma, \alpha}-\bar{\epsilon}\right)\right]}\right)$.

\section{RESULTS OF THE MEAN-FIELD APPROXIMATION}

\section{A. System with periodic boundary conditions}

First we briefly discuss the MFT for the infinite system with periodic boundary conditions. If we assume

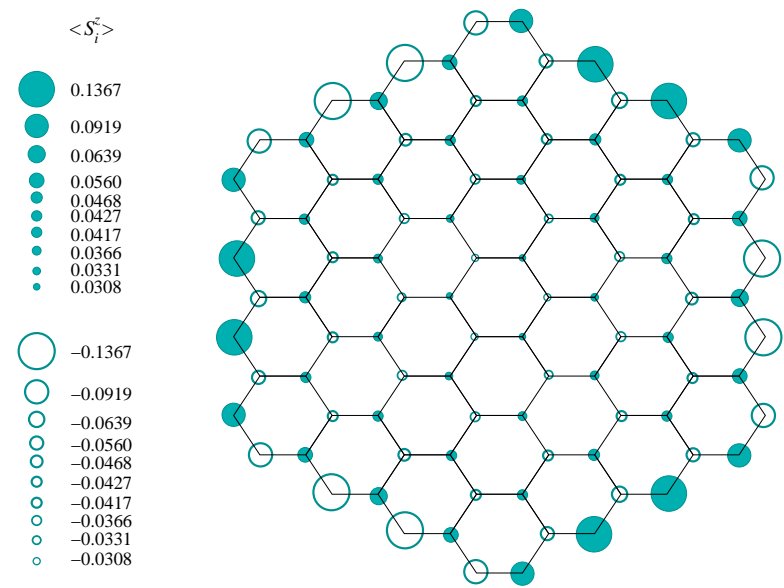

FIG. 1: (Color online) Mean-field result for the edge magnetization of a hexagonal graphene quantum dot with $N=96$ sites and zig-zag edges (at half filling and with $U=2 t$ ).

a Néel-ordered configuration, we find a Mott-Hubbard phase transition at the literature value $U_{c} \approx 2.23 t,{ }^{21}$ where the system goes from a paramagnetic semi-metal to an insulator with antiferromagnetic order. The asymptotic behavior of the Néel order parameter and the singleparticle gap is numerically consistent with linear behavior in $U-U_{c}$ for $U>U_{c}$, i.e., associated critical exponents equal to one. These unusual mean-field exponents reflect the unusual density of states ${ }^{21}$ which is linear close to the Fermi energy (compare also Ref. 23).

We use the critical value $U_{c}$ mainly to choose an approximate value of $U$ to describe graphene. In fact, the correct value of the Coulomb interaction in graphene is not yet known. Taking the value of $U$ in polyacetylene, where $U=10 \mathrm{eV}$ and $t=2.5 \mathrm{eV}$, suggests $U \approx 4 t$ for graphene. ${ }^{2}$ Since, at the MF level, this value locates the system well inside the antiferromagnetic phase and it is observed that large graphene sheets do not show magnetic order, we have decided to use a value of $U$ smaller than $U_{c}, U=2 t$ for the following computation.

\section{B. Edge magnetism on zig-zag edge}

It is well known that even for values of $U$ smaller than the critical value $U_{c}$, one observes a form of ferromagnetism on the zig-zag edge of a graphene ribbon ${ }^{9,13,16,17}$ or a quantum dot. ${ }^{14,15,19}$ As an example, Fig. 1 shows our results for the local magnetization of a hexagonal dot with 96 sites. One observes local ferromagnetic behavior at each zig-zag edge. By contrast, systems with armchair edges do not show specific magnetic properties and follow an evolution closer to the one of a system with periodic boundary conditions. The difference between the two edges appears to be a consequence of the fact that in the zig-zag case only one sublattice is represented on the edge while in the armchair case both sublattices are present. Detailed explorations ${ }^{15,17}$ demonstrated that the ferro- 

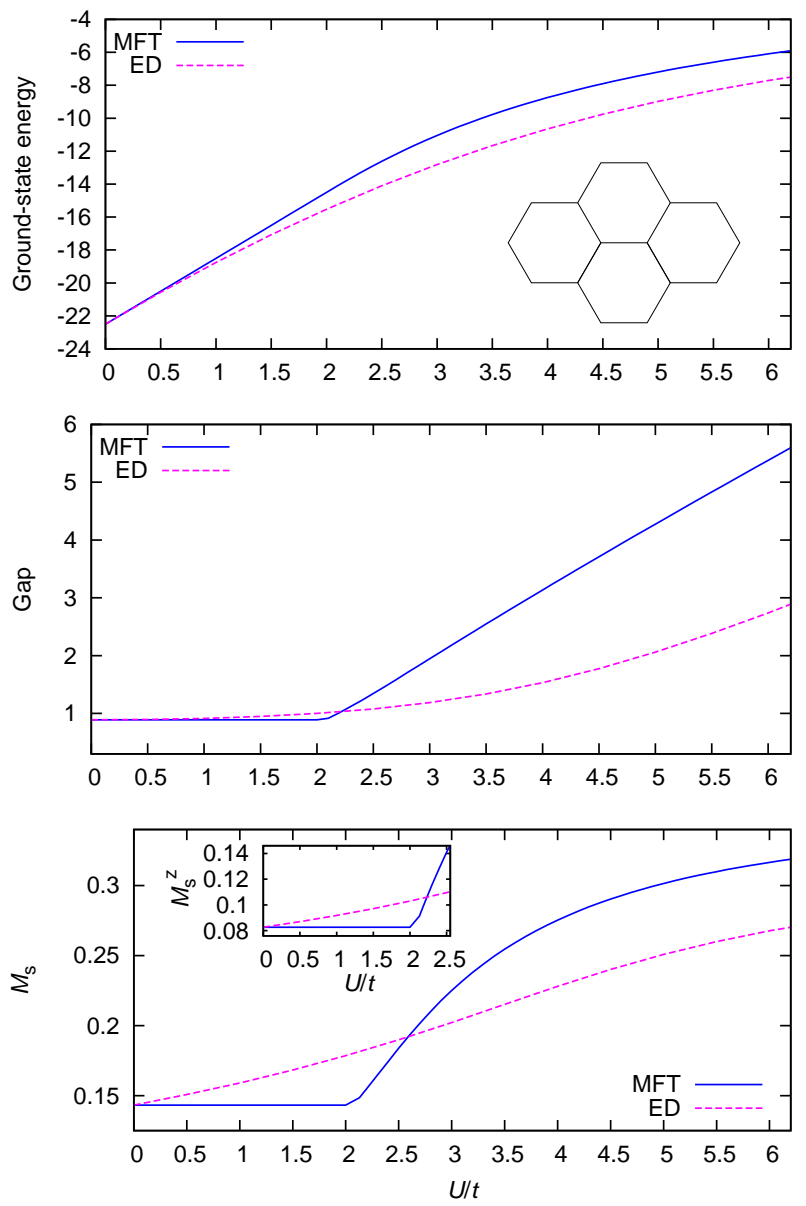

FIG. 2: (Color online) Comparison MFT-ED for the finitesize system of 16 sites sketched in the inset of the top panel at half filling. The bottom panel shows the total staggered magnetization $M_{\mathrm{s}}$ in the main panel and the $z$-component $M_{\mathrm{s}}^{z}$ in the inset.

magnetism of zig-zag edges resists to armchair defects and appears already for short edges.

\section{ACCURACY OF THE APPROXIMATION}

\section{A. Comparison with exact diagonalization for open boundary conditions}

To verify the accuracy of the MFT we first compare the results with those obtained with exact diagonalization (ED) of the Hubbard model which was performed with Spinpack. ${ }^{24}$ Due to the exponential growth of the Hilbert space, ED is limited to very small systems. Here we have studied the dot-like cluster of 16 sites shown in the inset of the top panel of Fig. 2. The following quantities were computed: (i) The ground-state energy. (ii) The charge gap defined as $\Delta E=E_{N-1}-2 E_{N}+E_{N+1}$, where $E_{n}$ is the ground-state energy in the sector with $n$ electrons. (iii) The $z$ - and total staggered magnetiza- tion as defined in terms of the longitudinal and total spin structure factor

$$
\begin{aligned}
M_{\mathrm{s}}^{z} & =\frac{1}{N} \sqrt{\sum_{i, j}(-1)^{i-j}\left\langle S_{i}^{z} S_{j}^{z}\right\rangle}, \\
M_{\mathrm{s}} & =\frac{1}{N} \sqrt{\sum_{i, j}(-1)^{i-j}\left\langle\vec{S}_{i} \cdot \vec{S}_{j}\right\rangle} .
\end{aligned}
$$

Here, $(-1)^{i-j}$ is a short-hand notation for $+1(-1)$ if $i$ and $j$ belong to the same (different) sublattice(s). Within MFT, the correlation functions appearing in (4.1) and (4.2) are computed from (2.5) and (2.6). In a numerical solution of the Hubbard model respecting SU(2) symmetry one finds $M_{\mathrm{s}}^{z}=M_{\mathrm{s}} / \sqrt{3}$.

Figure 2 shows a comparison of ground-state energy, charge gap, and the two staggered magnetizations computed both with MFT and ED at half filling, i.e., a total of 16 electrons. As expected, the two methods yield identical results for $U=0$ and the MF ground-state energy is always above the exact answer. The results for all three quantities stay close for $U \lesssim 2 t$. This supports the applicability of MFT at least as a semi-quantitative method in particular for the parameters of the dot shown in Fig. 1.

\section{B. Comparison to Quantum Monte Carlo for periodic boundary conditions}

In order to assess the quality of the MFT for larger but still finite systems, we employ QMC simulations. We use a projective determinantal QMC approach ${ }^{25}$ to obtain ground-state properties at half filling. Within this scheme, expectation values of a physical observable $A$ are obtained from

$$
\langle A\rangle=\lim _{\Theta \rightarrow \infty} \frac{\left\langle\Psi_{T}\left|e^{-\Theta H / 2} A e^{-\Theta H / 2}\right| \Psi_{T}\right\rangle}{\left\langle\Psi_{T}\left|e^{-\Theta H}\right| \Psi_{T}\right\rangle},
$$

where the trial wavefunction $\left|\Psi_{T}\right\rangle$ must be nonorthogonal to the ground state and $\Theta$ corresponds to a projection parameter. We found $\Theta=40 / t$ to be sufficient to obtain converged ground-state quantities within the statistical uncertainty. For the presented simulations, $\Theta$ was split into discrete step $\Delta \tau$ in the Trotter decomposition. We verified by extrapolating $\Delta \tau \rightarrow 0$ that taking $\Delta \tau=0.05 / t$ produced no discretization artifacts. The simulations were performed on systems with periodic boundary conditions.

Figure 3 shows our QMC and MFT data for a finite system with $N=162$ lattice sites. The top panel of Fig. 3 shows the energy per site. In the middle panel of Fig. 3 we compare the charge gap $\Delta E$ between MFT and QMC. In the QMC simulations the single-particle gap $\Delta_{\text {sp }}(\vec{k})$ was obtained by fitting the exponential tail of the imaginary-time displaced Green's function $G(\vec{k}, \tau) \propto$ $\exp \left(-\tau \Delta_{\mathrm{sp}}(\vec{k})\right)$ at large imaginary time $\tau$. The singleparticle gap $\Delta_{\mathrm{sp}}$ shown in Fig. 3 equals $\Delta_{\mathrm{sp}}(K)$, i.e., 

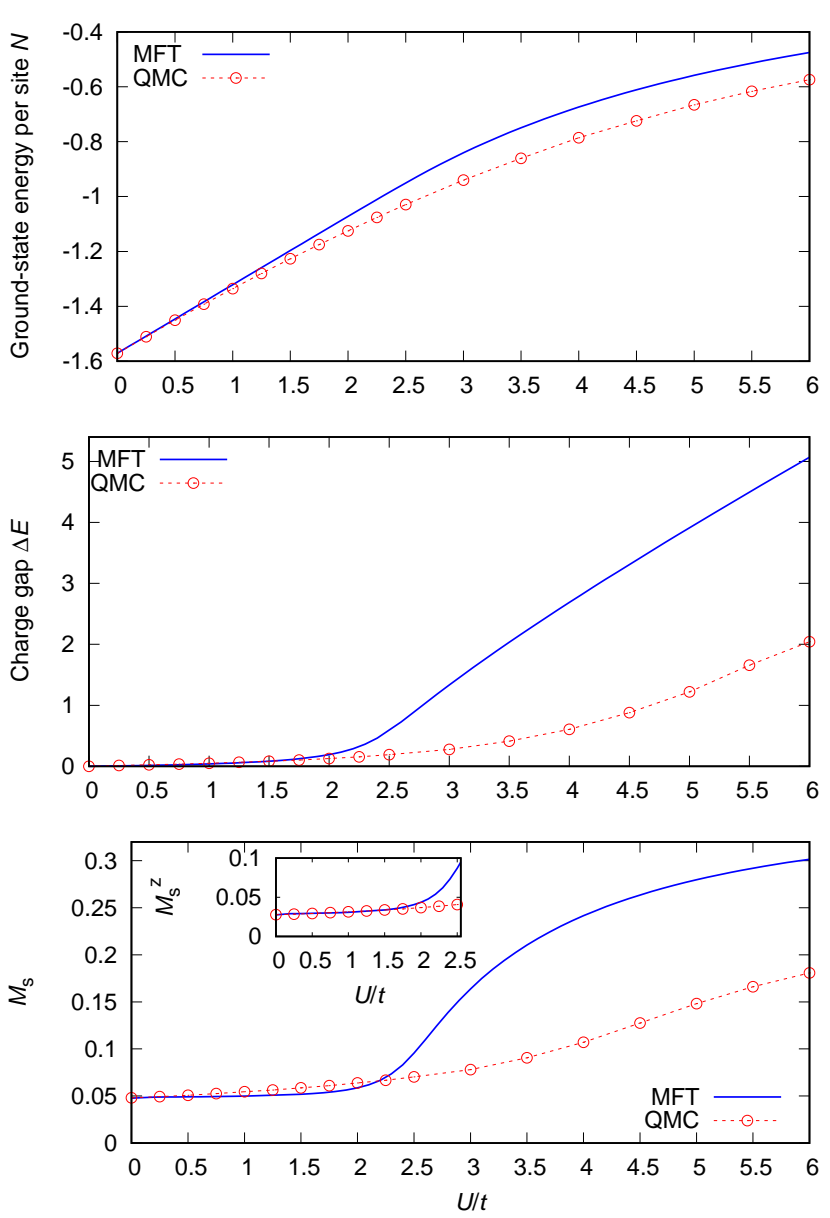

FIG. 3: (Color online) Comparison MFT-QMC for a system with periodic boundary conditions and $N=162$ sites at half filling. QMC error bars are smaller than the size of the symbols.

the smallest excitation gap at the Dirac points. Since the system under study is a particle-hole symmetric halffilled system, the chemical potential is right in the middle of the charge gap such that the charge gap is in fact twice the single-particle gap $\Delta E=2 \Delta_{\mathrm{sp}}$. Finally, the bottom panel of Fig. 3 compares the QMC results for the the total staggered magnetization Eq. (4.2) (main panel) and the $z$-component Eq. (4.1) (inset) with the MFT results.

Again, the MFT follows the QMC results closely for $U \lesssim 2 t$. In the present case, the MF curves for the energy, charge gap $\Delta E$, and $M_{\mathrm{s}}^{z}$ are always above the QMC curves. In fact, one observes that in the regime $U \lesssim 2 t$ the agreement between MFT and QMC is a bit better for $M_{\mathrm{s}}^{z}$ than for $M_{\mathrm{s}}$. This can be attributed to the MF approximation explicitly breaking the $\mathrm{SU}(2)$ symmetry. Indeed, in this case one finds that the MF contribution of the $x$ - and $y$-components to $M_{\mathrm{s}}$ are independent of $U$ for $U>0$ whereas the $z$-component increases with increasing $U$.

Appreciable quantitative differences can be observed in Fig. 3 at large $U$ in particular in $\Delta E$ and $M_{\mathrm{s}}$. Indeed,
MFT is known to underestimate the stability-range of the paramagnetic semi-metal by about a factor $2,^{21,26}$ i.e., quantitative differences are expected for $U$ larger than the mean-field critical value $U_{c} \approx 2.23 t$. Furthermore, in the limit $U \rightarrow \infty$ we expect to recover the $S=1 / 2$ Heisenberg model where it is known (compare, e.g., Refs. $27,28)$ that a full quantum mechanical treatment of the quantity defined in Eq. (4.2) yields a value which for large $N$ is only about $55 \%$ of the classical (i.e., MF) value $M_{\mathrm{s}}=1 / 2$. The difference at the right boundary of the bottom panel of Fig. 3 is indeed of this order. While the MFT and QMC deviate in the precise position of the quantum critical point, they agree on locating the system in the paramagnetic semi-metallic phase for $U \leq U_{c} \approx 2.23 t$ and the correspondence is at least semiquantitative for a finite-size system and $U \lesssim 2 t$.

\section{CONCLUSION AND PERSPECTIVES}

We investigated a self-consistent mean-field approximation to the Hubbard model on the honeycomb lattice, concentrating on half filling. The infinite system exhibits a Mott-Hubbard transition from a paramagnetic semimetal for a Coulomb repulsion $U<U_{c}$ to an antiferromagnetic insulator for $U>U_{c}$ with a MF critical value $U_{c} \approx 2.23 t .{ }^{21}$ The mean-field critical exponents associated to the gap and Néel order parameter are numerically consistent with the value one. ${ }^{21}$

We studied the accuracy of the MFT for finite-size systems with complementary exact diagonalization and quantum Monte Carlo simulations of the Hubbard model. We computed the ground-state energy, the single-particle gap, and the staggered magnetizations obtained from the spin correlation functions. The MFT reproduces the qualitative behavior found by the other two methods. Furthermore, the quantitative agreement is reasonable for $U \lesssim 2 t$, i.e., the region which is identified as a paramagnetic semi-metal both by MFT and QMC. For large values of $U$, quantitative differences become appreciable. However, the latter regime corresponds to an antiferromagnetic insulator, not relevant to graphene.

A weak-coupling instability to a canted antiferromagnet emerges in graphene when an in-plane magnetic field is turned on. ${ }^{29}$ This weak-coupling instability can be captured at the mean-field level and is confirmed by QMC simulations. ${ }^{29}$ Being equally a weak-coupling phenomenon, we believe that it will be possible to observe edge ferromagnetism in future QMC simulations with zigzag boundary conditions. Nevertheless, the MFT has access to bigger systems than QMC since it is numerically less demanding. Here we have presented the first piece of evidence that the MFT may be expected to be quantitatively reliable for $U \lesssim 2 t$. 


\section{Acknowledgments}

We wish to thank T. C. Lang for fruitful discussions, NIC Jülich and HLRS Stuttgart for the allocation of computer time, and the ESF for financial support through the INSTANS program. A.H. acknowledges support by the Deutsche Forschungsgemeinschaft through grant HO 2325/4-1 and S.W. and Z.Y.M. through grant WE $3649 / 2-1$.
1 K. S. Novoselov, A. K. Geim, S. V. Morozov, D. Jiang, Y. Zhang, S. V. Dubonos, I. V. Grigorieva, and A. A. Firsov, Science 306, 666 (2004).

2 A. H. Castro Neto, F. Guinea, N. M. R. Peres, K. S. Novoselov, and A. K. Geim, Rev. Mod. Phys. 81, 109 (2009).

3 L. A. Ponomarenko, F. Schedin, M. I. Katsnelson, R. Yang, E. W. Hill, K. S. Novoselov, and A. K. Geim, Science 320, 356 (2008).

4 B. Wunsch, T. Stauber, and F. Guinea, Phys. Rev. B 77, 035316 (2008).

${ }^{5}$ C. Stampfer, J. Güttinger, F. Molitor, D. Graf, T. Ihn, and K. Ensslin, Appl. Phys. Lett. 92, 012102 (2008).

${ }^{6}$ K. Nakada, M. Fujita, G. Dresselhaus, and M. S. Dresselhaus, Phys. Rev. B 54, 17954 (1996).

7 L. Brey and H. A. Fertig, Phys. Rev. B 73, 235411 (2006).

8 E. V. Castro, N. M. R. Peres, J. M. B. Lopes dos Santos, A. H. Castro Neto, and F. Guinea, Phys. Rev. Lett. 100, 026802 (2008).

9 M. Fujita, K. Wakabayashi, K. Nakada, and K. Kusakabe, J. Phys. Soc. Jpn. 65, 1920 (1996).

10 K. Wakabayashi, M. Fujita, H. Ajiki, and M. Sigrist, Phys. Rev. B 59, 8271 (1999).

11 T. Hikihara, X. Hu, H.-H. Lin, and C.-Y. Mou, Phys. Rev. B 68, 035432 (2003).

12 H. Lee, Y.-W. Son, N. Park, S. Han, and J. Yu, Phys. Rev. B 72, 174431 (2005).

13 Y.-W. Son, M. L. Cohen, and S. G. Louie, Phys. Rev. Lett. 97, 216803 (2006).

14 J. Fernández-Rossier and J. J. Palacios, Phys. Rev. Lett.
99, 177204 (2007).

15 S. Bhowmick and V. B. Shenoy, J. Chem. Phys. 128, 244717 (2008).

16 E. V. Castro, N. M. R. Peres, and J. M. B. Lopes Dos Santos, Journal of Optoelectronics and Advanced Materials 10, 1716 (2008).

17 H. Kumazaki and D. S. Hirashima, J. Phys. Soc. Jpn. 77, 044705 (2008).

18 J. J. Palacios, J. Fernández-Rossier, and L. Brey, Phys. Rev. B 77, 195428 (2008).

19 J. Viana-Gomes, V. M. Pereira, and N. M. R. Peres, Phys. Rev. B 80, 245436 (2009).

${ }^{20}$ O. V. Yazyev and M. I. Katsnelson, Phys. Rev. Lett. 100, 047209 (2008).

21 S. Sorella and E. Tosatti, Europhys. Lett. 19, 699 (1992).

22 D. C. Cabra and G. L. Rossini, Phys. Rev. B 69, 184425 (2004).

23 I. F. Herbut, V. Juričić, and B. Roy, Phys. Rev. B 79, 085116 (2009).

24 J. Schulenburg, URL http://www-e.uni-magdeburg.de/jschulen/spi

25 F. F. Assaad and H. G. Evertz, Lect. Notes Phys. 739, 277 (2008).

26 N. Furukawa, J. Phys. Soc. Jpn. 70, 1483 (2001).

27 J. Richter, J. Schulenburg, and A. Honecker, Lect. Notes Phys. 645, 85 (2004).

28 E. V. Castro, N. M. R. Peres, K. S. D. Beach, and A. W. Sandvik, Phys. Rev. B 73, 054422 (2006).

29 M. Bercx, T. C. Lang, and F. F. Assaad, Phys. Rev. B 80, 045412 (2009). 